\section{Radiojodtherapie ebnet Weg für andere Tumoren}

Die Heilungschancen bei differenzierten Schilddrüsenkarzinomen sind sehr gut. Allerdings werden diese Patienten im Laufe der nächsten 20 Jahre häufiger an einem anderen Tumor erkranken. Unter Verdacht steht die Radiojodtherapie.

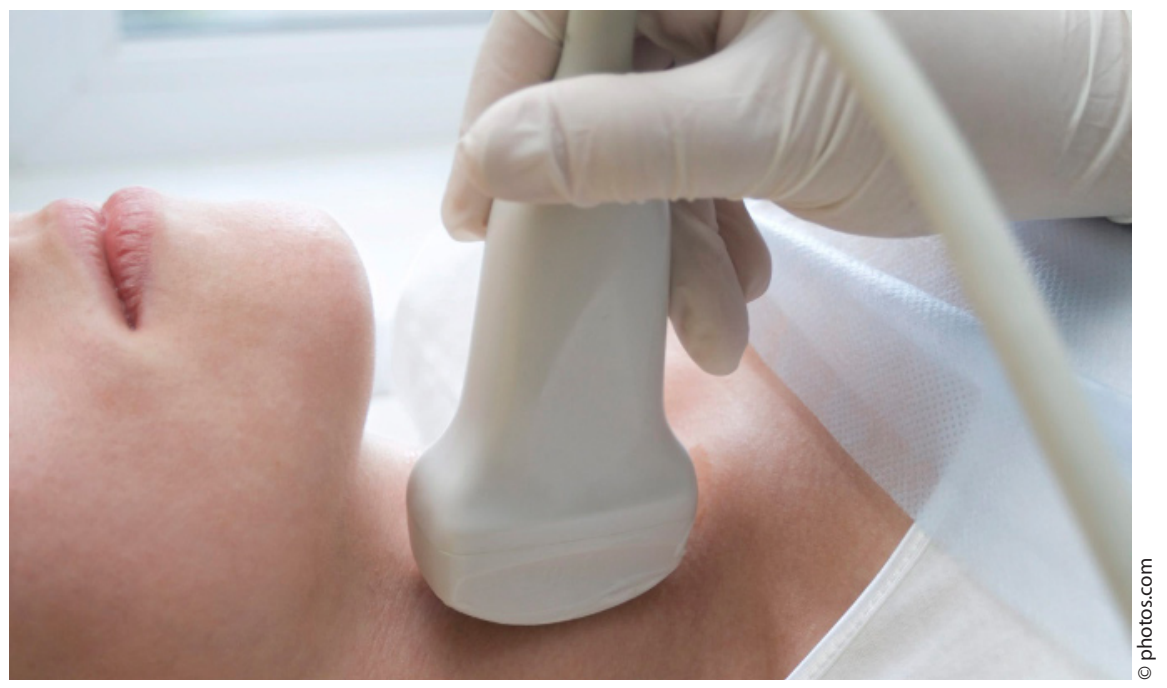

Wird ein Schilddrüsenkrebs diagnostiziert, sollte gut abgewogen werden, ob eine Radiojodtherapie notwendig ist.

W er ein differenziertes Schilddrüsenkarzinom (DTC) besiegt hat, hat ein erhöhtes Risiko für andere Tumorerkrankungen. Und nicht nur das: Die Heilungschancen sind dann auch schlechter. Ursache ist die Therapie des Schilddrüsenkarzinoms, speziell mit Radiojod.

Brian Hung-Hin Lang und Kollegen werteten die Daten von 1.122 Patienten aus, die zwischen 1971 und 2009 wegen eines DTC an der Universität Hongkong behandelt worden waren. Ausschlusskriterien waren eine frühere Radiojodtherapie sowie eine adjuvante lokale Bestrahlung. Von den verbleibenden 895 Patienten waren 634 mit radioaktivem Jod behandelt worden, die übrigen 252 nicht.

Nach median 95,3 Monaten musste bei insgesamt 64 Personen erneut die Diagnose Krebs in einem anderen Organ gestellt werden: Der Großteil dieser Patienten $(\mathrm{n}=56)$ hatte wegen des Schilddrüsentumors radioaktives Jod erhalten.

Auch beim kumulativen 20-JahresKrebsrisiko schnitten die mit Radiojod Therapierten signifikant schlechter ab $(13,5$ vs. $3,1 \%$; $=0,015)$. In einer multivariaten Analyse war die Radiojodthera- pie von allen untersuchten Variablen der einzige unabhängige Risikofaktor für einen späteren Krebs.

Die Bedeutung des radioaktiven Jods unterstreicht auch der Vergleich mit der Normalbevölkerung: Hier fiel die Krebsinzidenz nämlich nur bei den mit Radiojod Therapierten signifikant höher aus. Dabei scheint sich eine Radiojodtherapie für Frauen besonders ungünstig zu auszuwirken (SIR [standardisierte Inzidenz Ratio] 1,54 bei Frauen mit Radiojod vs. 0,92 bei Frauen ohne Radiojod).

Fazit: Auch wenn Jod-131 vor allem in die normalen oder entarteten Zellen der Schilddrüse eingelagert wird, reichert es sich offenbar auch in anderen Organen an, mit entsprechenden Konsequenzen für das Entartungsrisiko. Bei Schilddrüsenkarzinomen sollte daher die Indikation für eine Behandlung mit ionisierendem Jod vorsichtiger gestellt werden, insbesondere bei weniger gefährlichen Tumoren.

Dagmar Kraus

Hung-Hin B et al. Risk of second primary malignancy in differentiated thyroid carcinoma treated with radioactive iodine therapy. Surgery. 2012 Feb 14. [Epub ahead of print].

\section{Sommer, Sonne, trockene Nase?}

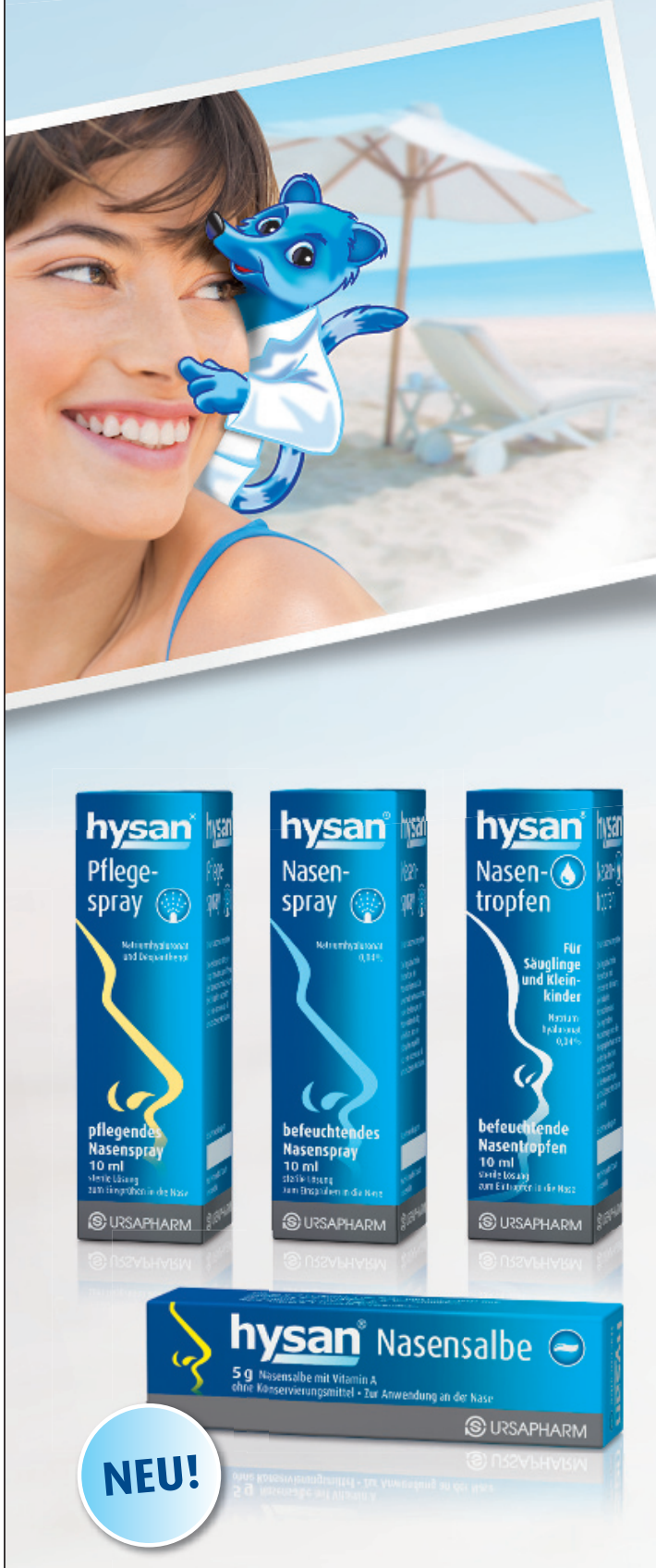

- bei trockenen und gereizten Nasenschleimhäuten

- effektive Pflege, lang anhaltende Befeuchtung

- exklusiv in der Apotheke erhältlich

URSAPHARM Arzneimittel GmbH

Industriestraße 35 · 66129 Saarbrücken · www.ursapharm.de 